\title{
Initiative targets malaria eradication
}

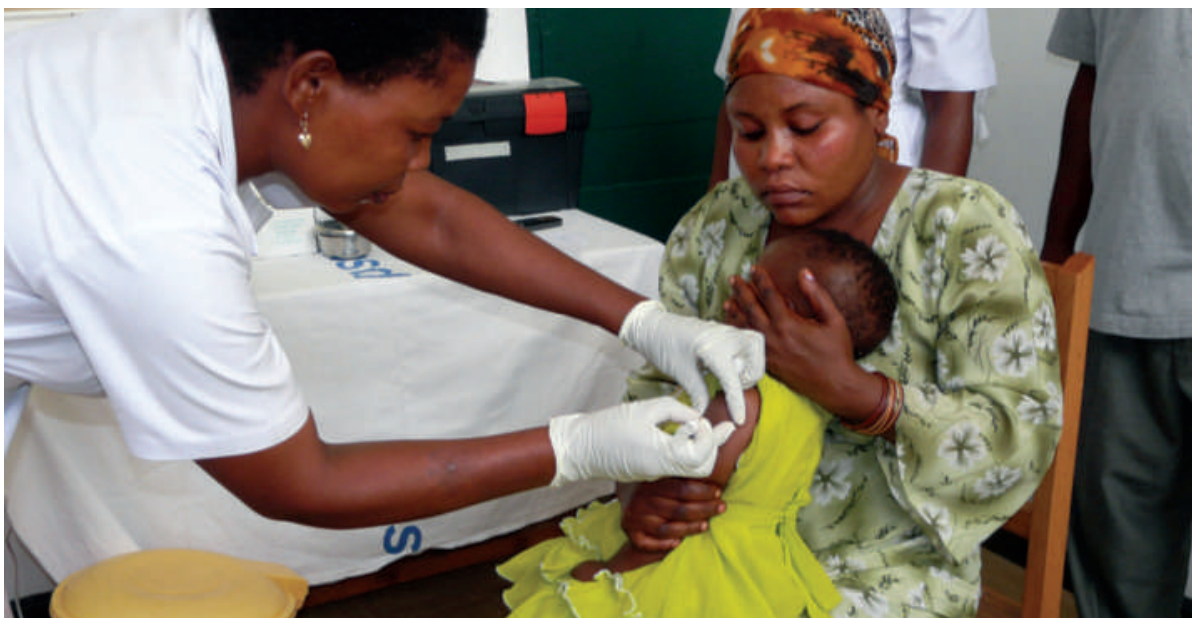

Next-generation vaccines are intended to move beyond the initial infection phase.

The PATH Malaria Vaccine Initiative (MVI), the main public-private partnership for developing vaccines against the disease, this week announced a major overhaul of the sorts of vaccine candidates it will support in the future, as well as the ways in which it will select them.

The move follows a 2007 call by Bill and Melinda Gates - major funders of malaria research - for researchers to not just control the disease, but aim to eradicate the parasite. "Whether you believe that eradication is attainable or not, it has prompted substantial discussion in the malaria community as to what tools would be needed to reach it," says Robert Sinden, a malaria biologist at Imperial College London.

Until now, clinical efforts towards a vaccine have focused on on the individual level by reducing cases and deaths. But the MVI, based in Bethesda, Maryland, will now also invest heavily in vaccines that block transmission of the malaria parasite. These vaccines provide no direct benefit to individuals but might fight the disease at the population level.

Transmission-blocking vaccines generate antibodies to the stage of the parasite that replicates sexually in the mosquito gut. So when a mosquito takes blood from an infected person who has been vaccinated, it will also suck up antibodies and immune cells that destroy the parasites as they replicate. Laboratory tests and pilot studies in humans have shown that such vaccinations drastically lower the numbers of infected mosquitoes.

"I'm delighted that the MVI is finally plugging this gap in its portfolio," says Adrian Hill, a malaria-vaccine researcher at the University of Oxford, UK.

Another type of vaccine targeted for increased support is live-attenuated vaccines of the Plasmodium sporozoite - the stage of the parasite that initially infects the liver. For decades, the logistics of making a working vaccine using sporozoites have been considered insurmountable; among other things it requires manually dissecting billions of sporozoites in mosquito farms.

Sanaria, a company in Rockville,

Maryland, run by veteran malaria researcher Stephen Hoffman, took up the sporozoite challenge six years ago, and in April it began a phase I clinical trial of its radiation-attenuated vaccine. "A lot of people were sceptical about that, and I think a lot of credit should go to Hoffman and his people at Sanaria [because] they have overcome that," says Anthony Fauci, director of the US National Institute of Allergy and Infectious Diseases. The trial will end next June.

Even if the trial is a success, however, the obstacles to scaling-up and delivering the vaccines remain formidable.

Nonetheless, Sanaria's progress has spurred the MVI to take the approach seriously. It also intends to support several labs that are developing sporozoites attenuated by knocking out genes. Although still at the research stage, this technique might be a more reliable way to attenuate the parasite than radiation, says Ashley Birkett, the
MVI's director of preclinical development. By 2025, the MVI aims to have nextgeneration vaccines that are more than $80 \%$ effective and that last for at least four years. The most effective first-generation effort in its portfolio, GlaxoSmithKline Biologicals' RTS,S vaccine - which targets the initial infection phase in humans - falls far short of that. Phase II trials have suggested that it cuts infections and severe disease by around $50 \%$, enough to save many lives, although the duration of the protective effect remains uncertain. A phase III trial is under way at 11 sites in Africa.

Combining RTS,S with vaccines targeted at other stages of the malaria parasite's life cycle could produce much stronger effects. Animal studies suggest that combined vaccines show a substantial multiplier effect, says Hill, whose T-cell vaccines are a current favourite for the first trials with RTS,S.

The MVI intends to pursue only combined vaccine trials in future.

Moreover, the RTS,S results have set a higher bar for contenders, says Birkett. In the future, he says, vaccine candidates will need to pass much more extensive preclinical testing, and initial testing in a few human volunteers, with only the highly effective ones being taken forward. Such early testing systems have been chronically underfunded.

One approach that is proving powerful is human challenge experiments, in which a few volunteers are vaccinated and their immune responses closely studied. Such studies should be the rule, says Hill. "Nothing should be taken forward into Africa without taken forward into Africa without knowing knowing it does something." Birkett says that
the MVI is supporting a dedicated Human Challenge Center at the Seattle Biomedical Research Institute in Washington, one of only four such centres worldwide, that will open next year.

As well as weeding out potential vaccine candidates, the MVI intends to look at funding partnerships beyond the malariavaccine community to find feedstock for its pipeline. "Of course the malaria community will remain our core community," says Birkett, "but there's a lot of untapped expertise out there, including experts in HIV, influenza, cancer and biotechs who have different vaccine strategies."

Declan Butler 